\title{
Histoire courte en quelques brefs chapitres
}

Chers membres de la FMH,

Le 30 juin, lorsque la comptabilité du Secrétariat général retirera définitivement mon nom de la liste des salaires, j'aurai été au service de la FMH exactement cinq ans et dix mois. Bref laps de temps ou longue période? Comment mesurer le temps? En mois? En jours? Ou même en minutes, comme dans le cadre du TARMED? Comment l'évaluer au moment du calcul? Donner plus de poids aux cinq premières minutes? Que valent les cinq dernières minutes? Qu'est-ce qui compte, au fond?

Je tente une rétrospective.

\section{1}

C'est l'année du $100^{e}$ anniversaire et j'arrive à la FMH en qualité de «secrétaire générale désignée», pour reprendre une fonction qui avait été assumée pendant des décennies «de cette façon et pas autrement». Je suis chargée de «moderniser les choses» conformément au mandat que l'on me remet d'un ton décidé mais dont le contenu reste vague. Un héritage plutôt complexe.

\section{2}

correspond à ma première grande tâche, celle d'organiser une votation générale, en quelque sorte le baptême du feu. 2002 est l'année du «grand changement»: les accords bilatéraux avec l'UE entrent en vigueur en abolissant «l'obligation» qui existait de facto de s'affilier à la FMH. J'ai le mandat peu réjouissant d'élaborer des scénarios de redimensionnement plus ou moins radicaux pour le cas d'un exode massif des membres, alors que la réalité, ou plus précisément le Conseil fédéral, nous joue un tour. Au lieu de l'exode attendu, le blocage de l'accès à la pratique privée entraîne une augmentation du nombre de membres et au lieu de l'indignation redoutée, malgré l'augmentation des taxes, il provoque un flot de demandes de titres. Du jamais vu. Il en va ainsi lorsqu'on planifie: les choses se passent de façon encore plus imprévue.

\section{3}

est marqué par le recensement de la valeur intrinsèque. Une vague de mauvaise humeur s'abat sur le Secrétariat général tel un raz-de-marée, se brisant sur les pare-feu de nos serveurs de messagerie électronique et submergeant les lignes téléphoniques. Le bureau de la valeur intrinsèque croule sous les caisses postales débordant de documents dont le classement prendra des mois. Ce recensement met à rude épreuve les capacités et la solidarité de notre système qui, fort heureusement, se verra libéré d'une tâche encore plus ingrate. En effet, il ne sera pas nécessaire de lancer le référendum sur la levée de l'obligation de contracter: le Parlement a pitié de nous et renonce à ébranler une nouvelle fois nos structures.

\section{4}

continue gaiement sur cette lancée. Après des d'années d'affûtage et de mise au point, alors que l'on ne sait finalement plus comment il fonctionne ni même s'il fonctionne, le TARMED est introduit. Plus ou moins sous forme expérimentale, dans le cadre de l'activité courante: mais cela fonctionne. Et la FMH se donne un nouveau président.

\section{5}

voit ce dernier lancer la réforme des structures. Pas question, une fois de plus, de couler des jours tranquilles au sein de l'appareil administratif.

\section{6}

conduit à l'avènement de la réforme des structures en nous lançant un nouveau défi, celui de la mettre en œuvre (règlement d'exécution, etc.).

\section{7}

est maintenant l'année où l'on apprend à vivre autrement, en cherchant ses marques dans un environnement recomposé. Le voyage à Jérusalem se poursuit. Qui décrochera une place, qui partira?

\section{5,8 ans...}

... et un nombre croissant d'anglicismes à l'ordre du jour: «SwissDRG», «Medical Education», «Managed Care», «Health Professional Card». Mon rôle dans tous ces domaines a été celui du catalyseur. Aider et libérer la voie pour que les spécialistes puissent faire leur travail. Je fus en partie aussi l'initiatrice ou l'accélérateur. Je ne me suis jamais vue comme contrôleuse ou commandante. En revanche comme rénovatrice: j'ai revu à fond le règlement $\mathrm{du}$ personnel, conjointement avec les cadres, pour en faire une œuvre progressiste équilibrant, à un niveau élevé, les responsabilités et les compétences. La comptabilité de la FMH a été mise à jour, les systèmes informatiques nécessaires à la gestion ont été installés ou affinés, le processus de budgétisation a été complètement remanié. Le rapport d'activité a été modernisé et, de long article hétéroclite publié dans le Bulletin des médecins suisses, il est devenu une «revue bleue» avec des chiffres et des faits.

\section{0 mois...}

Les réorganisations sont aussi allées bon train à l'interne: des services ont été regroupés ou transférés, de nouveaux départements ont été créés, avec des noms exotiques comme «DDQ» ou «eHealth». Le service tarifaire a déménagé d'Oberkirch à Olten, la vague de rénovation a touché non seulement le site internet de la FMH, mais 
également l'immeuble du Secrétariat général, à l'Elfenstrasse, qui s'est vu doté après 30 ans d'une nouvelle cafétéria, d'une nouvelle réception et de bureaux fraîchement peints. Sans parler du tout nouveau support à vélos ...

\section{9 jours ...}

Tant de personnes arrivées et parties durant ce temps: 54 nouveaux arrivants au sein du personnel, dont 21 nous ont déjà quittés. 42 sorties au total, dont 7 départs à la retraite et 1 décès. Changement de 5 membres du Comité central et du président. Un nombre de membres qui a passé de 28938 à 33418.

Que signifient ces chiffres? Que reste-t-il au bout du compte?

- La FMH: elle n'est pas une «quantité négligeable», mais pas non plus une institution incontournable. Elle doit trouver son chemin entre tempête et élan vers l'avenir, entre renouvellement et pérennité.

- Les membres: si leur nombre évolue, leurs qualités ne changent pas. Leur volonté d'être représentés de manière appropriée, de disposer de services utiles et de règlements mesurés demeure intacte tout comme leur hétérogénéité et la contradiction, qui en découle, consistant parfois à vouloir le beurre et l'argent du beurre.

- Le Comité central: il naviguera toujours entre opposition et rapprochement et continuera à explorer les notions de «collégialité» et de «responsabilité».

- Les collaboratrices et collaborateurs du Secrétariat général: ils ne sont ni des employés derrière un guichet ni un collectif que l'on pourrait manœuvrer à sa guise. Ils méritent du respect, une ligne claire et des critiques constructives.

- Cinq ans et dix mois, ou le «cas Ami Müller», première femme à la tête du Secrétariat général de la FMH.

Ce qui restera, c'est un morceau d'histoire. L'avenir est, selon Armin Nasehi, «un chemin inconnu auquel on ne peut pas se dérober».

Je vous souhaite tout le meilleur!

Annamaria Müller Imboden 